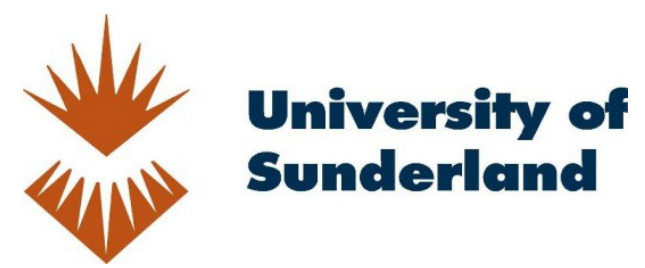

Yeates, Giles, Rowberry, Michelle, Dunne, Stephen, Goshawk, Michelle, Mahadevan, Mythreyi, Tyerman, Ruth, Salter, Mandy, Hillier, Martin, Berry, Alister and Tyerman, Andy (2016) Social cognition and executive functioning predictors of supervisors' appraisal of interpersonal behaviour in the workplace following acquired brain injury. NeuroRehabilitation, 38 (3). pp. 299-310. ISSN 1053-8135

Downloaded from: http://sure.sunderland.ac.uk/id/eprint/9376/ 
Please refer to the usage guidelines at http://sure.sunderland.ac.uk/policies.html or alternatively contact sure@sunderland.ac.uk. 


\title{
Social cognition and executive functioning predictors of supervisors' appraisal of interpersonal behaviour in the workplace following acquired brain injury
}

\author{
Giles Yeates ${ }^{\mathrm{a}, *}$, Michelle Rowberry ${ }^{\mathrm{b}}$, Stephen Dunne ${ }^{\mathrm{b}}$, Michelle Goshawk ${ }^{\mathrm{b}}$, Mythreyi Mahadevan ${ }^{\mathrm{a}}$, \\ Ruth Tyerman ${ }^{\mathrm{a}}$, Mandy Salter ${ }^{\mathrm{a}}$, Martin Hillier ${ }^{\mathrm{a}}$, Alister Berry ${ }^{\mathrm{b}}$ and Andy Tyerman ${ }^{\mathrm{a}}$ \\ ${ }^{a}$ Working Out Programme, Community Head Injury Service, Buckinghamshire Healthcare NHS Trust, UK \\ ${ }^{\mathrm{b}}$ Momentum Skills Brain Injury Vocational Rehabilitation, UK
}

\begin{abstract}
.
BACKGROUND: Social cognition and executive functioning difficulties following acquired brain injury have been linked to negative employment outcomes, such as demotion and loss of vocational roles. These are very counter-intuitive and challenging difficulties for other employees and work supervisors who have little or no brain injury knowledge, whose perceptions of play a key role in their responses to these difficulties and the final outcome of such problems for vocational status.

OBJECTIVES: This study aimed to study the relationship between social cognition and executive functioning difficulties and the perceptions of work supervisors' appraisal of survivor interpersonal behaviour and social skills in the workplace.

METHOD: The performance of 73 survivors of acquired brain injury (47\% TBI, 38\% CVA, 15\% other ABI type; $73 \%$ male; mean age 45.44 years, range 19-64 years; mean time since injury 6.36 years, range 10.5-31.33 years), currently in a vocational rehabilitation placement) on neuropsychological tests of executive functioning and social cognition was measured. Informant ratings on the Social Skills Factor subscale from the Work Personality Profile (WPP, Bolton \& Roessler, 1986) were used as the primary outcome measure, a vocational functioning questionnaire assessing social and presentational aspects of workplace behaviour. The raters were non-clinical workplace informants acting in a supervisory role (supervisory placement providers and job coaches).

RESULTS: Correlational analysis identified significant associations between the WPP and survivor goal-orientated planning and implementation, mentalising ability, recognition of positive and negative emotions, and recognition of simple sarcasm (all significant at $p<0.05$ ). These correlates were entered into a stepwise multiple regression. The combination final of survivor mentalising ability and executive functioning explained $32 \%$ of the variance in the WPP ratings $(\mathrm{F}(2,52)=12.15, p<0.001)$. CONCLUSION: Certain limitations of the study withstanding, the current findings add to previous literature in highlighting the relevance of survivor executive functioning and social cognition difficulties for the perceptions and appraisal of work colleagues, consistent with other studies that have identified negative vocational outcomes associated with such neuropsychological difficulties. The implications for vocational rehabilitation are discussed.
\end{abstract}

Keywords: Brain injury, stroke, social cognition, mentalising, executive functioning, vocational, work, interpersonal, social skills

*Address for correspondence: Dr. Giles Yeates, Community Head Injury Service, Buckinghamshire Healthcare NHS Trust, Camborne Centre, Jansel Square, Aylesbury, Bucks, HP217ET,
UK. Tel.: +441296337760; Fax: +441296337743; E-mail: Giles.Yeates@buckshealthcare.nhs.uk. 


\section{Background}

\subsection{Social cognition and executive difficulties in the workplace post-injury}

The psychosocial consequences of both executive functioning and social cognition difficulties are now accepted as significant and enduring, with negative outcomes associated with such deficits including marital relationship quality (Blonder et al., 2012; Yeates, 2013), and community integration (Knox \& Douglas, 2009; Struchen et al., 2008, 2011). A return to a vocational role, either paid or voluntary is a desirable rehabilitation outcome. It provides an answer to the often posed question "what do you do then?" It can be a marker of continuity if there is a return to a pre-injury role and/or the possibility of a new meaningful opportunity if it is a transition from unemployment and inactivity to vocational engagement. As such a vocational role is a vital building-block within the ongoing task of identity reconstruction post-injury (Walsh et al., 2014). A range of psychological, social and financial gains for survivors and relatives follow from a successful resumption of vocational activity (Mills \& Kreutzer, 2015; Tyerman, Tyerman \& Viney, 2008).

However injury-related changes themselves have been shown to challenge the return to vocational activity. Injury severity has been identified as a predictor of vocational status five years postinjury (Wehman et al., 1993). Furthermore, specific domains of cognitive impairments have been identified as barriers to work post-injury. Predictors of negative vocational outcomes (demotion, reduced vocational status from pre-injury roles and unemployment) include executive dysfunction (Crepeau \& Sherzer, 1993; Nybo et al., 2004; Vilkki et al., 1994), social communication impairments (Power \& Hershenson, 2003). The latter include difficulties in turn-taking and regulating quantity of verbal output (Watt \& Penn, 2000), emotion-recognition and social communication (Knox \& Douglas, 2009, Struchen et al., 2011), overt inappropriate behaviours such as irritability (Groswasser et al., 1999; Wehman et al., 1993), and other changes described at the level of personality (Brooks et al., 1987). These predictors have been demonstrated alongside mental health status post-injury (Hoofen et al., 2001) and a lack of awareness of disability (e.g., Sherer et al., 1998; Watt \& Penn, 2000). Struchen and colleagues (2008) found that a combination of social communication and executive function ability in survivors predicted a significant amount of variance in an occupational functioning measure, beyond demographic and other injury-related variables.

These constellations of difficulties present a challenge to the rehabilitation clinician, equipped with a knowledge and experience of brain injury sequelae. The world of work is a step away from this specialist knowledge, with colleagues and managers often having little or no brain injury-related knowledge. Instead of a rehabilitation or neuropsychological framework, work-staff are likely to make sense of instances of executive and social communication difficulties through a different set of referents, beliefs and expectations. These are often based on a language of productivity and diligence, the right kind of work ethic and attitude, being a team player, trustworthiness, and harassment and prejudice.

\subsection{Vocational rehabilitation response to interpersonal challenges in the workplace}

Furthermore, instances of such difficulties are likely to be significant challenges to work supervisors and colleagues. To someone without brain injury knowledge, exposure to these problems must be a confusing experience. A survivor may be functioning inconsistently over time and situations. There might be a disconnection between what a survivor is saying about their performance and what would actually be happening. In other instances these encounters may be uncomfortable or even hurtful for employees. Colleagues may feel intimidated, awkward or offended by the comments or behaviour of some survivors during a coffee break (Bowen, Yeates \& Palmer, 2010). It becomes clear that when comparing instances of these difficulties with the work-place corporate language and belief systems mentioned above, there is a far less than ideal fit between the two, unlikely to promote understanding and management. A line manager, while trying to be understanding and accommodating to a survivor's needs in the workplace, would also be organised by a duty of care to non-injured employees and so be limited in the support and opportunity that can continue to beprovided to a survivor over time.

While a key element of vocational brain injury rehabilitation is the liaison between clinicians, job coaches/vocational specialists and employers or placement providers, it is rare for such staff to be present constantly alongside the survivor while in the work environment. The impact of executive and social communication difficulties will therefore be 
constructed through the eyes and frameworks of work colleagues at the moment they occur. Even rehabilitation efforts to forewarn work personnel about such challenges and support the sense-making and responses of employers have been described as limited (Bowen, Yeates \& Palmer, 2010).

\subsection{Meaning-making social systems in the workplace}

Therefore the perceptions, belief systems and language of employers, work colleagues and vocational placement providers remain significant, the psychosocial context within which the aforementioned neuropsychological difficulties occur during survivors' vocational activity. Outside of neurorehabilitation, there is an established tradition of systems/cybernetics/social constructionist approaches to work organisations (Campbell, Coldicott \& Kinsella, 1994). That is, the understanding of how meanings and roles are constructed within the active social processes of the work organisation and the use of this understanding to guide supportive interventions that are congruent with the idiosyncratic meanings and social structures of the given organisation. This approach may offer value to vocational rehabilitation, and has been tentatively explored by Bowen and colleagues (2010) and Bowen, Yeates and Palmer (2010). Within this approach, the understanding and management of the impact of executive and social communication difficulties in the workplace would require a simultaneous focus on the unique ways in which such difficulties are interpreted and accorded meaning by work colleagues, and the resultant social processes that may ensue from such interpretations. The latter would likely have a bearing on vocational outcome for the survivor.

The aforementioned research linking executive and social communication difficulties with negative vocational outcomes can be developed through process research identifying mediating/moderating factors and likely causal paths between survivor injury/adjustment-related variables and eventual vocational outcomes. The perceptions and belief systems of work colleagues is likely to be one such critical process variable.

\subsection{Research strategies to elucidate workplace appraisals of interpersonal behaviour}

A pluralist methodological approach to this topic would is advisable. A qualitative investigation of the actual language, beliefs and frameworks used in the accounts of employers/workcolleagues in their sense making of survivor neuropsychological difficulties would yield rich information. Sale and colleagues (1991) adopted a qualitative approach in their interviews of employers, and report that "interpersonal difficulties", "social cue misperceptions" and "inappropriate verbalisations" were the most common causes of job-separations for traumatic brain injury survivors. In their interviews of both workers and supervisors working alongside survivors, Meulenbroek and colleagues (2016) identified a range of communication skills necessary for optimal vocational functioning, including social communication. Further qualitative work is needed that is focused on the language used by employers in making sense of these and related difficulties.

This approach would be complemented by quantitative paradigms aiming to identify generalizable inter-relationships between the critical variables discussed above. This study adopts such an approach, a quantitative investigation of the interrelationships between survivor neuropsychological functioning (considered to be stable, 18 months plus post-injury) and work supervisors' perceptions of survivor social skills and interpersonal behaviour, using employment terms of reference rather than clinical rehabilitation language. This approach is consistent with the theme of the special issue as a whole, as the workplace following brain injury is considered to be a unique relational context. In this setting the meanings and appraisals formed within constituent social relationships are assumed to be significantly different from those formed in the rehabilitation and family/home contexts. The discernment of significant interrelationships between these variables would be valuable in targeting vocational rehabilitation efforts aimed at maximising employment outcome, by both supporting survivors' impairments through compensatory strategies and responding to the perceptions of employers and work colleagues.

Based on the existing literature, it is hypothesised that measures of survivor social cognition will predict supervisors' appraisals of workplace interpersonal behaviour. In addition, the existing demonstrated social outcomes from executive dysfunction in survivors in previous studies led to the hypothesis that executive functioning measures will have an additional association with the primary outcome measure. However it is hypothesised that less variance in the outcome will be predicted by an executive functioning measure, as this association is considered to be indirect. 


\section{Method}

\subsection{Participants}

\subsubsection{Brain injury survivors}

73 survivors of moderate-severe acquired brain injury (at least 1.5 years post-injury) participating in vocational rehabilitation (across three rehabilitation sites in the UK). Demographics and injury-related data are presented in Table $1.73 \%$ were male, $27 \%$ female. Traumatic brain injury survivors made up the majority of the sample (47\%), followed by cerebrovascular accident (ischaemic and haemorrhagic, $38 \%$ ). Other ABI subtypes in the remaining category included hypoxia, infection and post-surgical tumour resection.

As the sample was recruited from vocational rehabilitation programmes, survivors did not demonstrate a level of communication, cognitive or behavioural problems that would preclude their participation in community and vocational activity. As such they are also typical of the survivor subgroup who will attempt to return back to work post-injury. Survivors were either participating in, or had just completed a minimum 3 months or ongoing work trial. These were in charities or other 3rd sector organisations or a return to a pre-injury employing organisation but in a protected role with fewer responsibilities.

\subsubsection{Vocational supervisors}

These were purposively selected as non-clinicians, employing a supervisory workplace perspective on behaviour and productivity rather than a clinical focus

Table 1

Sample descriptive data

\begin{tabular}{lll}
\hline Gender & Male & 53 \\
& Female & 20 \\
\hline Age & Mean & 45.44 years \\
& SD & 10.56 \\
Injury Type & Range & $19-64$ \\
& TBI & 34 \\
& CVA ischaemic & 16 \\
& CVA Haemorrhagic & 12 \\
& Hypoxia & 3 \\
& Encephalitis & 4 \\
Injury Severity & Tumour Resection & 1 \\
(Length Post Traumatic & Other & 3 \\
Amnesia) & Mean & 3.88 weeks \\
& SD & 4.0 \\
Time Post-Injury & Range & \\
& Mean & $0.5-15$ \\
& SD & 6.36 years \\
& Range & 6.28 \\
& & $1.5-31.33$ \\
\hline
\end{tabular}

on neurological injury, cognitive impairments and mental health. 33 informants made the ratings using the outcome measure of the 73 survivor participants. These informants were either job coaches $(57 \%)$, or work placement providers $(43 \%)$. The former acted in a bridging role between the clinical team in the vocational rehabilitation services and the workplace, identifying, establishing and maintaining work placements, and acting as the main point of liaison with the employer.

\subsection{Procedure and measures}

All survivor participants completed a battery of neuropsychological tests and self-report mood questionnaires. At the same time vocational supervisors completed the Work Personality Profile (Bolton \& Roessler, 1986), to enable a cross-sectional correlational analysis.

\subsubsection{Primary outcome measure - Work Personality Profile}

The Work Personality Profile (WPP, Bolton \& Roessler, 1986) has been developed and validated for use in vocational settings, particularly in sheltered work and rehabilitation contexts. It is available as a self-report measure with a parallel version designed to be completed by a vocational informant who rates a client's vocational performance in areas relating to personality, motivation and interpersonal behaviour as applied to work. The WPP has 58 items, rated 1-4, with higher scores indicating greater proficiency (and $\mathrm{x}$ for no opportunity to observe the behaviour). A range of subscale scores are calculated: 5 factor-derived scales and 11 'rationallyderived scales'. Those items specifically relating to interpersonal behaviour were of interest in this study, and accordingly the factor analysis-derived Social Skills Factor subscale was extracted as this study's main outcome measure. This subscale is comprised of 12 items, including: "shows interest in what others are doing".... "seeks out co-workers to be friends"... . "appears comfortable in social interactions"... "supportive of others in group tasks"... "offers assistance to co-workers when appropriate".. "is sought out by co-workers". The WPP Social Skills subscale has an internal consistency of 0.83 and inter-rater reliabilities of 0.35 to 0.44 . The WPP has acceptable convergent validity with measures of vocational aptitude and adequately predicts vocational outcome for respondents undergoing vocational training (Bolton, 1985). 


\subsubsection{Survivor executive functioning}

All survivors were tested on neuropsychological measures of executive functioning and social cognition measures (at 10.5 years post-injury or longer) as part of their clinical pathway and this data was accessed for this study. As such, the executive functioning measures were selected for either their ecological validity or the significance of the underlying construct for vocational outcome as identified in previous studies. The tests included measures of goal-directed formulation and implementation of plans: the Zoo Map and Modified 6 Elements subtest from the Behavioural Assessment of Dysexecutive Syndrome (Wilson et al., 1996). Addition tests were measures of initiation of novel verbal responses (Letter Fluency subtest from the Delis-Kaplan Executive Function System, Delis, Kaplan \& Kramer, 2001), inhibition of automatic verbal responses (Hayling Sentence Completion Test, Burgess \& Shallice, 1997) and spatial anticipation/cognitive flexibility (Brixton Spatial Awareness Test, Burgess \& Shallice, 1997).

These executive tests have adequate reliability coefficients (Letter Fluency reliability coefficients have been reported as ranging from 0.7 to 0.9 , Abwender et al., 2001; Hayling: split-half $0.8-$ 0.93 ; test-retest $0.52-0.76$; Brixton: split-half 0.62 ; test-retest 0.71). The BADS subtests are designed for their novelty on first participation so test-retest statistics are inappropriate. All executive measures demonstrate adequate convergent validity with other measures of executive function (Burgess \& Shallice, 1997; Wilson et al., 1996). A case has been made for the ecological validity of many of these tests (Burgess et al., 1997), and survivor scores on the two BADS subtests correlate with ratings of dysexecutive problems in everyday life made by significant others (Wilson et al., 1996).

\subsubsection{Survivor social cognition}

These measures included tests of mentalising/theory of mind, the ability to infer the intentions and perspectives of others. These measures werebased static visual/facial stimuli (Reading the Mind in the Eyes Test, Baron-Cohen et al., 2001) and on story vignettes (Recognition of Faux Pas Test, Stone et al., 1998). For the latter the standard total score ut of 40 for the 10 faux pas stories were used, in addition to two supplemental scores that categorise different types of mentalising error, first order errors where the impact on the victims of the faux pas was not detected, and intentionality errors where the naïve intention of the unwitting perpetrator of the faux pas (question 4 "why did they do it?" for each story) was not recognised (for details on this scoring procedure, see Yeates and colleagues, 2013). Mentalising has been identified as an essential component of wider forms of social inference, such as the detection of differing forms of sarcasm, sincerity and deceit. These abilities were tested using the video social scenario stimuli in parts 2 and 3 from The Awareness of Social Inference Test (TASIT, McDonald et al., 2003).

Additional social cognition abilities included video-based test of emotion recognition was used (Emotion Evaluation Test, Part 1 of the TASIT, McDonald et al., 2003), alongside a vignette-based task of social judgement-making, including the detection of violations of social norms (Social Situations Task, Dewey, 1991). The final social cognition test was a gamble task paradigm of emotion-based decision-making (Bangor Gambling Task, Bowman \& Turnbull, 2004), the use of gut-feeling to guide decision-making in ambiguous scenarios.

In terms of psychometric properties, reliability estimates for the TASIT subtests ranged from 0.7 to 0.9 and correlations with other social cognition, information processing and executive functioning tests are in line with theoretical assumptions of the interrelationships between these neuropsychological constructs (McDonald et al., 2006a). The ecological validity of the TASIT has been demonstrated via significant correlations between test performance and everyday social-conversational abilities (McDonald et al., 2006). The two mentalising tasks used in this study have been shown to converge with other measures of mentalising (Baron-Cohen et al., 2003; Stone et al., 1998) and test performance predicts aspects of interpersonal and social functioning, as rated by others (Milders et al., 2001). No reliability information has been published for the Social Situations Task or Bangor Gambling Task. Njomboro, Humphreys and Deb (2014) discerned ABI survivors from controls using the social situations task. Bowman and Turnbull (2004) demonstrated adequate convergent validity between the Bangor Gamble Task and the Iowa Gambling Task (Bechara et al., 1994), and similar sensitivity to lesions of the neuro-anatomical substrate hypothesised to mediate emotion-based decision-making.

\subsubsection{Survivor emotional functioning}

As the relevance of TBI survivor emotional status to vocational outcome has been demonstrated by van der Horn and colleagues (2013), this was opera- 
tionalised as variable to be controlled for in this study. Survivor emotional functioning was assessed using self-report questionnaires of anxiety and depression (Hospital Anxiety \& Depression Scale, HADS, Zigmond \& Snaith, 1983)and post-traumatic stress (Impact of Events Scale-Revised, Weiss \& Marmar, 1997), both with acceptable psychometric properties and widely used within ABI samples in previous research.

\subsection{Analysis}

The measures were entered into blocks of correlational analyses with the WPP, grouped by classification: demographics, mood, executive functioning, mentalising and social inference, with a final block comprising emotion recognition, social judgements and emotion-based decision-making. Those correlations that were significant at $p<0.05$ were then entered into a hierarchical multiple regression with WPP ratings as the dependent variable. The risks against making a type I error given the large number of variables were considered to be low as a) the associations between similar neuropsychological and vocational variables of interest have been demonstrated in previous studies and b) in the final regression analysis the use of the more conservative computer-generated stepwise variable entry method with the significant correlates will further reduce the risk of type I error.

\section{Results}

\subsection{Descriptive analysis}

The means and standard deviations of survivors on all neuropsychological tests, mood questionnaires and their ratings by vocational informants on the Work Personality Profile Factor 2 subscale is summarised in Table 2 below. There were no significant differences in scores on measures between age categorisations, gender or injury subtype:

\subsection{Correlational analysis}

Pearson one-tailed correlation coefficients were calculated, following a priori assumptions of the directions of possible relationships. Scatterplots were examined to confirm that the assumptions of the test were met. There were no significant associations between the continuous data in the demographics, injury-related and mood-self report data and the WPP scores.

Tables 3-5 below present the results of correlational analyses of the relationship between the WPP SS scores and tests of mentalising and social inference; emotion recognition, social judgements and emotion-base decision-making; executive functioning.

In the mentalising and social inference block (Table 3), there were significant positive correlations between the WPP-SS and Faux Pas Test faux pas stories total score $(r=0.263 ; p<0.05)$, a significant negative correlation with Faux Pas Test First Order Errors Total score $(r=-0.387 ; p=0.001)$, and a significant positive correlation with TASIT Part 2 Simple Sarcasm Total Score $(r=0.273 ; p<0.05)$. In the next block of social cognition measures (Table 4), WPP-SS scores were significantly positively associated with Emotion Evaluation Test (EET) Total $(r=0.385 ; p=0.001)$, EET Positive Emotions Total $(r=0.298 ; p=<0.05)$, and EET Negative Emotions Total $(r=0.364 ; p<0.01)$ scores, in addition to the Number of correctly identified instances of social rule violations $(r=0.274 ; p<0.05)$ on the Social Situation Task. In the executive functioning block (Table 5), the only significant association was a positive correlation between the Modified 6 Elements Profile Score and the WPP $(r=0.312 ; p<0.05)$. All of the other associations from all of the aforementioned variables were non-significant.

\subsection{Regression analysis}

Initially, the demographic and injury-related measures were entered as predictors for each variable, to control for these factors. No significant results were found and the analysis proceeded to the next stage. The five significant neuropsychological correlates of the WPP scores from the previous analysis were entered into ahierarchical regression analysis, via the software's (SPSS) automatic stepwise procedure. This generated a first model where Faux Pas First Order Error score predicted $19 \%$ of the variance in WPP ratings $(\mathrm{F}(1,53)=11.83, p=0.001)$. A second model was then produced where a combination of Survivor Faux Pas First Order Error Scores and Modified Six Elements Profile Scores predicted $32 \%$ of the variance in the dependent measure $(F(2,52)=12.15, p<0.001)$. The full results of the regression analysis are summarised in Table 6 below. 
Table 2

Survivor and partner group data on all measures with reference normative data

\begin{tabular}{lcc}
\hline Measure & M (SD) & Range \\
\hline Survivor Vocational Interpersonal Functioning & & $1.08-4$ \\
Work Personality Profile Social Skills Factor & $3.27(0.71)$ & $0-18$ \\
Survivor Mood & & $0-17$ \\
HADS Anxiety & $6.65(4.2)$ & $0-110$ \\
HADS Depression & $35.63(26.87)$ & \\
IES-R & & $14-33$ \\
Mentalising/Social Inference Measures & $24.01(4.46)$ & $13-39$ \\
Reading Mind in the Eyes & $27.63(6.29)$ & $0-16$ \\
Faux Pas FP Stories Total & $5.03(3.66)$ & $0-9$ \\
Faux Pas FP 1st Order Error Total & $3.87(2.25)$ & $4-20$ \\
Faux Pas 2nd Order Error Total & $15.47(3.98)$ & $5-20$ \\
TASIT P2 Sincere & $16.86(3.66)$ & $9-20$ \\
TASIT P2 Simple Sarcasm & $17.62(2.52)$ & $13-32$ \\
TASIT P2 Paradoxical Sarcasm & $26.08(3.59)$ & $12-37$ \\
TASIT P3 Lies & $24.57(4.42)$ & \\
TASIT P3 Sarcasm & & $9-28$ \\
Other Social Cognition Tests & $21.78(4.18)$ & $1-12$ \\
TASIT P1 EET Total & $9.01(2.18)$ & $4-16$ \\
TASIT P1 EET + VE Total & $12.77(2.64)$ & $5-12$ \\
TASIT P1 EET - VE Total & $9.69(1.97)$ & $-78-56$ \\
Social Situations Violation Total & $-2.79(26.64)$ & \\
BGT No Good-Bad Choices Total & & $0-4$ \\
Executive Functioning & $2.44(1.32)$ & $0-4$ \\
BADS Zoo Map Profile & $3.08(1.06)$ & $1-8$ \\
BADS Modified 6 Elements Profile & $5.13(1.42)$ & $1-10$ \\
Hayling Overall Sten Score & $5.65(2.27)$ & $1-18$ \\
Brixton Total Sten Score & $9.0(3.86)$ & \\
D-KEFS Letter Fluency & & \\
WPP Work Personality Profil HADS Hospital Anxiety & \\
\hline
\end{tabular}

WPP = Work Personality Profile; HADS = Hospital Anxiety Depression Scale; IES- $R=$ Impact of Event ScaleRevised; TASIT $=$ The Awareness of Social Inference Test Parts 1, 2 \& 3; EET = TASIT Part 1 Emotion Evaluation Test; BGT $=$ Bangor Gambling Task; BADS = Behavioural Assessment of Dysexecutive Syndrome; D-KEFS = Delis-Kaplan Executive Functioning System

Table 3

Correlations between work personality profile - social skills factor subscale and survivor tests of mentalising and social inference

\begin{tabular}{|c|c|c|c|c|c|c|c|c|c|}
\hline Variable & 1 & 2 & 3 & 4 & 5 & 6 & 7 & 8 & \\
\hline 1. Work Personality Profile-SS & 1 & & & & & & & & \\
\hline \multirow[t]{2}{*}{ 2. Reading the Mind in the Eyes } & 0.215 & 1 & & & & & & & \\
\hline & 0.070 & & & & & & & & \\
\hline \multirow[t]{2}{*}{ 3. Faux Pas Total FP Stories } & $0.267^{*}$ & $0.289^{* *}$ & 1 & & & & & & \\
\hline & 0.027 & 0.008 & & & & & & & \\
\hline \multirow[t]{3}{*}{ 4. Faux Pas 1st Order Error Total } & $-0.387^{* *}$ & - & -0.751 & 1 & & & & & \\
\hline & 0.001 & $-0.319^{* *}$ & & & & & & & \\
\hline & & 0.994 & 0.000 & & & & & & \\
\hline \multirow[t]{2}{*}{ 5. Faux Pas Intentionality Error Total } & 0.000 & 0.089 & -0.093 & -0.293 & 1 & & & & \\
\hline & 0.999 & 0.424 & 0.400 & 0.051 & & & & & \\
\hline \multirow[t]{2}{*}{ 6. TASIT P2 Sincere Total } & -0.097 & 0.182 & -0.069 & 0.111 & -0.090 & 1 & & & \\
\hline & 0.436 & 0.110 & 0.547 & 0.341 & 0.440 & & & & \\
\hline \multirow[t]{2}{*}{ 7. TASIT P2 Simple Sarcasm Total } & 0.273 & 0.173 & $0.346^{* *}$ & -0.402 & 0.014 & -0.179 & 1 & & \\
\hline & 0.028 & 0.131 & 0.002 & 0.000 & 0.908 & 0.116 & & & \\
\hline \multirow[t]{2}{*}{ 8. TASIT P2 Paradoxical Sarcasm Total } & 0.163 & 0.175 & $0.339^{* *}$ & $-0.274^{*}$ & -0.082 & -0.049 & $0.432^{* *}$ & 1 & \\
\hline & 0.195 & 0.228 & 0.003 & 0.018 & 0.482 & 0.671 & 0.000 & & \\
\hline \multirow[t]{2}{*}{ 9. TASIT P3 Lie } & 0.090 & 0.285 & 0.102 & -0.070 & -0.176 & $0.333^{* *}$ & 0.045 & 0.176 & 1 \\
\hline & 0.477 & 0.012 & 0.378 & 0.551 & 0.130 & 0.003 & 0.698 & 0.127 & \\
\hline \multirow[t]{2}{*}{ 10. TASIT P3 Sarcasm } & 0.067 & $0.369^{* *}$ & 0.175 & -0.174 & -0.036 & 0.174 & $0.333^{* *}$ & $0.391^{* *} 0.347$ & $0.347 \quad 1$ \\
\hline & 0.598 & 0.000 & 0.128 & 0.135 & 0.759 & 0.129 & 0.003 & & 0.002 \\
\hline
\end{tabular}

${ }^{*} p<0.05 .{ }^{* *} p<0.01$. WPP $=$ Work Personality Profile; TASIT $=$ The Awareness of Social Inference Test Parts $1,2 \& 3$; EET $=$ TASIT Part 1 Emotion Evaluation Test. 
Table 4

Correlations between work personality profile - social skills factor subscale and survivor tests of emotion-recognition, social judgement-making and emotion-based decision-making

\begin{tabular}{|c|c|c|c|c|c|c|}
\hline Variable & 1 & 2 & 3 & 4 & 5 & 6 \\
\hline 1. Work Personality Profile-SS & 1 & & & & & \\
\hline \multirow[t]{2}{*}{ 2. EET TASIT Total } & $0.385^{* *}$ & 1 & & & & \\
\hline & 0.001 & & & & & \\
\hline \multirow[t]{2}{*}{ 3. EET TASIT + VE Emotions Total } & $0.298^{* *}$ & $0.772^{* *}$ & 1 & & & \\
\hline & 0.013 & 0.000 & & & & \\
\hline \multirow[t]{2}{*}{ 4. EET TASIT - VE Emotions Total } & $0.364^{* *}$ & $0.873^{* *}$ & $0.364^{* *}$ & 1 & & \\
\hline & 0.002 & 0.000 & 0.001 & & & \\
\hline \multirow[t]{2}{*}{ 5. Social Situations Task Identified Violations Total } & $0.274^{*}$ & 0.020 & -0.085 & 0.092 & 1 & \\
\hline & 0.024 & 0.762 & 0.457 & 0.419 & & \\
\hline \multirow{2}{*}{ 6. BGT No Goo-Bad Choices Total } & -0.033 & -0.010 & -0.084 & 0.050 & 0.193 & 1 \\
\hline & 0.793 & 0.931 & 0.457 & 0.659 & 0.092 & \\
\hline
\end{tabular}

${ }^{*} p<0.05,{ }^{* *} p<0.01 . \mathrm{WPP}=$ Work Personality Profile; TASIT $=$ The Awareness of Social Inference Test Parts 1,2 \& 3; EET $=$ TASIT Part 1 Emotion Evaluation Test; BGT = Bangor Gambling Task.

Table 5

Correlations between work personality profile - social skills factor subscale and survivor tests of executive functioning

\begin{tabular}{|c|c|c|c|c|c|c|}
\hline Variable & 1 & 2 & 3 & 4 & 5 & 6 \\
\hline 1. Work Personality Profile-SS & 1 & & & & & \\
\hline 2. BADS Zoo Map Profile & $\begin{array}{c}0.076 \\
536\end{array}$ & 1 & & & & \\
\hline \multirow[t]{2}{*}{ 3. BADS Modified 6 Elements Profile } & 0.312 & 0.138 & 1 & & & \\
\hline & $0.014^{*}$ & 0.246 & & & & \\
\hline \multirow[t]{2}{*}{ 4. Hayling } & 0.088 & 0.143 & 0.162 & 1 & & \\
\hline & 0.478 & 0.211 & 0.175 & & & \\
\hline \multirow[t]{2}{*}{ 5. Brixton } & -0.011 & $0.409^{* *}$ & 0.143 & $0.234^{*}$ & 1 & \\
\hline & 0.928 & 0.000 & 0.237 & 0.038 & & \\
\hline \multirow[t]{2}{*}{ 6. D-KEFS Letter Fluency } & 0.263 & 0.313 & -0.066 & 0.253 & 0.111 & 1 \\
\hline & 0.057 & $0.019^{*}$ & 0.656 & 0.063 & 0.427 & \\
\hline
\end{tabular}

${ }^{*} p<0.05^{* *} p<0.01$. WPP $=$ Work Personality Profile; BADS = Behavioural Assessment of Dysexecutive Syndrome; DKEFS $=$ Delis-Kaplan Executive Functioning System.

Table 6

Summary of stepwise multiple regression analysis for variables predicting workplace social skills (work personality profile social skills factor), as rated by vocational informants

\begin{tabular}{|c|c|c|c|c|c|c|}
\hline \multirow[b]{2}{*}{ Variable } & \multicolumn{3}{|c|}{ Model 1} & \multicolumn{3}{|c|}{ Model 2} \\
\hline & $B$ & $S E B$ & $B$ & $B$ & $S E B$ & $\beta$ \\
\hline Faux Pa Test 1st Order Errors & -0.082 & 0.024 & $-0.427^{* *}$ & -0.078 & 0.022 & -0.404 \\
\hline Modified Six Elements & & & & 0.251 & 0.078 & $0.370^{* *}$ \\
\hline$R^{2}$ & & 0.182 & & & 0.319 & \\
\hline F for change in $R^{2}$ & & $11.83^{* *}$ & & & $12.15^{\text {** }}$ & \\
\hline
\end{tabular}

${ }^{* *} p<0.01$.

\section{Conclusions}

\subsection{Study findings and limitations}

This study adds to previous findings in highlighting the significance of survivorsocial cognition and executive functioning for the work environment, in particular mentalising and goal-orientated plan formulation and implementation. The respective contribution of the two variables in explaining the variance in workplace appraisals of survivor inter- personal behaviour were in line with our hypotheses (that survivor social cognition functioning would have a greater association with the outcome variable). However, the fact that the goal-directed planning/implementation executive functioning variable demonstrated a greater predictive association than a range of other social cognition variables was counter to our general hypotheses. Based on the variables operatioanlised in this study, these findings can act to refine our understanding of the relationships between survivor social cognition, executive 
functioning and work supervisor appraisals of interpersonal behaviour specifically.

That is, it is mentalising rather than emotion recognition, other forms of social inference, social judgment making and emotion-based decision-making that seems critical for supervisors' perceptions of social behaviour in the workplace. Furthermore, it is mentalising in combination with planning and organising that has the greatest bearing on others' perception of work-relevant social skills and behaviours. In the unique social context of the workplace, the ability to represent the perspectives of others based on the situation and their behaviour, together with the ability to formulate and implement plans of action in response to multiple goals, has the greatest fit with the intrinsic and unique social aspects of work environments. There will of course however be considerable variation across work roles and settings. These aspects include more formal social rules and conventions, the need to negotiate multiple and differing social roles within one setting (e.g., CEO, supervisor, colleagues known well or otherwise, and customers (whom may vary in their perspectives and needs). These elements occur alongside the need to attend to task-related goals, environmental distractions and other non-social information.

While not contributing to the predictive regression equation, the significant correlations with sarcasm detection, emotion recognition and social rule violation detection also suggest their relevance to workplace interpersonal functioning. These correlates would not be taken forward in the planned analysis if a Bonferoni correction was applied to compensate for the large number of comparisons (which would yield a threshold significance level of $p<0.01$ for each correlate). However these variables are consistent with some of the findings of social cognition abilities relevant to the workplace reported in previous studies (Knox \& Douglas, 2009, Struchen et al., 2011).

An additional perspective is that the majority of the variance in the outcome measure has not been explained by the independent variables operationalised in this study. Workplaces and the social appraisals formed within them are complex contexts with multiple influences and processes. It is also likely that task demands and roles unique to specific work situations, varying across job types will determine the impact of specific survivor social cognition and executive functioning abilities.

The novel contribution of this investigation is to employ an outcome measure sensitive to the lan- guage and frames of reference common to the work place when an individual's personality, behaviour and conduct as an influence on other colleagues is appraised and evaluated. Indeed it is interesting that both mentalising (the ability to infer the intentions and perspectives of others) planning/organising and have a combined predictive relationship with vocational informants' ratings of social skills. This shows both the social relevance of each function (and in combination, such as difficulties holding others in mind when struggling to manage multiple, concurrent goals) but also the independence of work supervisor categorisations from neuropsychology functioning typologies. For example, it is plausible that difficulties in planning and organising can be perceived by work colleagues as disrespectful and inconsiderate of the needs and experiences of others. It is argued here that the vocational informant perspective is the most relevant and enduring frame of reference against which survivor neuropsychological impairments and emotional difficulties are understood and accorded meaning. That is, more enduring than the time-limited perceptions and responses of vocational rehabilitation specialists and clinicians.

Major limitations of this study are that the survivors in the sample were in vocational placements in liaison with a brain injury rehabilitation service, and the work supervisors were inevitably influenced to a greater or larger degree by this perspective. The level of association varied from job coaches as the closest in position to clinicians, and placement provider key contacts as the furthest away in perspective, but still in liaison with the vocational team. As such the educational and supportive influence of the clinicians cannot be ignored and this demarcates a possible significant difference from the perspective of employers and work colleagues who have no contact with a vocational brain injury rehabilitation service. The latter group would be harder to sample, and as noted in the introduction, vocational placements do still struggle and even fail despite vocational rehabilitation specialist intervention. It is assumedtherefore that the data and conclusions reported here do have some generalisable value to the workplace following brain injury as a whole. This requires further confirmatory validation in future studies that are able to investigate other workplace informant samples.

\subsection{Recommendations for future research}

The associations demonstrated in this crosssectional study require replication and further con- 
firmation in subsequent studies. Future quantitative research is required using prospective longitudinal and time-series designs to track trajectories of workplace interpersonal ratings over-time following a baseline period of neuropsychological assessment, and in addition identify any additional mediating or moderating influences of other situational variables. This will substantiate assumptions of causality from the associations identified in the current crosssectional study. Additional research goals would be to investigate the predictive utility of other measures of social cognition and executive functioning in relation to workplace social skills. The latter outcome variable may also be more sensitively explored using an alternative quantitative measure from the occupational psychology literature, hitherto unidentified in brain injury research. Finally, as mentioned in the background section, a parallel research strategy would be the use of qualitative methodology to interview those connected to survivors of brain injury in the workplace. The rich details and nuances of how survivor neuropsychological difficulties in social cognition and executive function are identified, constructed and accorded meaning or importance within differing work settings and roles could be sensitively explored within a qualitative paradigm.

\subsection{Implications for vocational rehabilitation}

The limitations of this data withstanding, this study cumulatively adds to its predecessors in making a case for social cognition difficulties to be a focus of vocational rehabilitation, consistent with recent calls to support and develop the emergence of positive psychological skills in the workplace (Mills \& Kreutzer, 2015). The data reported here signpost to the trial of both cognition rehabilitation strategies for the survivor, and intensive liaison with employers/placement providers. With regards to the former, those social cognition rehabilitation approaches that aim to teach specific emotion recognition, mentalising and social problem-solving skills in a scripted, controlled manner (e.g., Bornhofen \& McDonald, 2008; for an overview of strategies, see Yeates, 2014), are most likely to be useful in guiding survivors in customer-facing roles. These and certain in interactions with colleagues which are predictable, controlled and guided by a protocol would be most amenable to these interventions. These strategies direct the survivor to attend to certain aspects of facial expression, posture or voice in others. Alternatively/in addition, survivors are prompted to conjecture at underlying perspectives, intentions and other mental states of others, perhaps in response to the survivor's own behaviour (e.g., the T-ScEmo intervention reported by Spikman and colleagues, 2013). The findings above also suggest the possible social value of executive strategies such as Goal Management Training (Robertson, 1996) and the use of checklists, pagers and alerts (Burke et al., 1991; Manly et al., 2004) in the workplace.

It is arguably those social interactions characterised by subtle and changing norms that are harder to support (e.g., moderating conduct with colleagues based on changing familiarity, changing background context such as on-task versus during break-time, but still discerning the difference between a colleague and a friend). These moments require on-line, responsive and flexible socio-emotional processes. Perhaps the use of a social communication partner, as advocated by Leanne Togher and colleagues (2004; this issue) may confer a unique role in these vocational settings, where safe online feedback can be provided, and appropriate responses prompted as a survivor negotiates the social dimension of their workplace.

Finally, all of these survivor-focused intervention approaches need to be implemented alongside concurrent support offered to the employer and work colleagues. Ongoing liaison with the vocational rehabilitation team, providing education of social cognition difficulties ahead of their emergence in the workplace, and offering varied and responsive points of contact for those in the workplace will contribute to the creation of conditions for open, supportive conversations and timely interventions. However the likelihood of employers to raise the occurrence of social transgressions in a timely manner is still not guaranteed with such supportive measures in place.

\section{Conflict of interest}

None to declare.

\section{References}

Abwender, D. A., Swan, J. G., Bowerman, J. T., \& Connolly, S. W. (2001). Qualitative analysis of verbal fluency output: Review and comparison of several scoring methods. Assessment, 8 , 323-336.

Baron-Cohen, S., Wheelwright, S., Hill, J., Raste, Y., \& Plumb, I. (2001). The "Reading the Mind in the Eyes" Test revised version: A study with normal adults, and adults with Asperger syndrome or high-functioning autism. The Journal of Child 
Psychology and Psychiatry and Allied Disciplines, 42, 241251.

Bechara, A., Damasio, A. R., Damasio, H., \& Anderson, S. W. (1994), Insensitivity to future consequences following damage to human prefrontal cortex. Cognition, 50, 7-15.

Blonder, L. Z., Pettigrew, L. C., \& Kryscio, R. J. (2012). Emotion recognition and marital satisfaction in stroke. Journal of Clinical \& Experimental Neuropsychology, 34(6), 634-642.

Bolton, B. (1985). Measurement in rehabilitation. In E. Pan, S. Newman, T. Backer., \& C. Vash (Eds), Annual Review of Rehabilitation(Vol 4). New York: Springer, pp. 115-144.

Bolton,B., \& Roessler, R. (1986). Manual for the work personality profile. Arkansas Research and Training Center in Vocational Rehabilitation, Arkansas.

Bowen, C., Hall, T., Newby, G., Walsh, B., Weatherhead, S., \& Yeates, G. (2009). The Impact of Brain Injury on Relationships Across the Lifespan and Across School, Family and Work Contexts. Human Systems: The Journal of Consultation and Training, 20, 65-80.

Bowen, C., Yeates, G. N., \& Palmer, S. (2010). A Relational Approach to Rehabilitation: Thinking about Relationships after Brain Injury. London: Karnac.

Bowman, C. H., \& Turnbull, O. H. (2004). Emotion-based learning on a simplified card game: The iowa and bangor gambling tasks. Brain \& Cognition, 55(2), 277-282.

Brooks, D. N., Campsie, L., Symington, C., Beattie, A., \& McKinlay, W. W. (1987). The effects of severe head injury on patient and relative within seven years of injury. Journal of Head Trauma Rehabilitation, 2, 1-13.

Burgess, P. W., Alderman, N., Evans, J., Emslie, H., \& Wilson, B. A. (1998). The ecologicalvalidity of tests of executive function. Journal of the International NeuropsychologicalSociety, $4,547-558$.

Burgess, P. W., \& Shallice, T. (1997). The Hayling and Brixton Tests. Bury St. Edmunds, UK: Thames Valley Test Company.

Burke, W. H., Zencius, A. H., Wesolowski, M. D., \& Doubleday, F. (1991). Improving executive function disorders in brain injured clients. Brain Injury, 5, 241-252.

Campbell, D., Coldicott, T., \& Kinsella, K. (1994). Systemic Work with Organisations. London: Karnac.

Crepeau, F., \& Sherzer, P. (1995). Predictors and indicators of work status after traumatic brain injury: A meta-analysis. NeuropsychologicalRehabilitation, 3, 5-35.

Delis, D. C., Kaplan, E., \& Kramer. J. H. (2001). Delis-Kaplan Executive Function System. San Antonio, TX: The Psychological Corporation.

Dewey, M. (1991). Living with Aspergers syndrome. In U. Frith. (Ed.), Autism and Aspergers syndrome. Cambridge: Cambridge University Press, pp. 184-206.

Groswasser, Z., Melamed, S., Agranov, E., \& Keren, O. (1999). Return to work as an integrative outcome measure following traumatic brain injury. Neuropsychological Rehabilitation, 9, 493-504.

Knox, L., \& Douglas, J. (2009). Long-term ability to interpret facial expression after traumatic brain injury and its relation to social integration. Brain \& Cognition, 69, 442-449

Manly, T., Heutink, J., Davison, B., Gaynord, B., Greenfield, E., Parr, A., Ridgeway, V., \& Robertson, I. H. (2004). An electronic knot in the handkerchief: 'Content free cueing' and the maintenance of attentive control. Neuropsychological Rehabilitation, 14(1-2), 89-116.
McDonald, S., Bornhofen, C., Shum, D., Long, E., Saunders, C., \& Neulinger, K. (2006). Reliability and validity of The Awareness of Social Inference Test (TASIT): A clinical test of social perception. Disability \& Rehabilitation, 28(24), 1529-1542.

McDonald, S., Flanagan, S., Martin, I., \& Saunders, C. (2004). The ecological validity of TASIT: A test of social perception. Neuropsychological Rehabilitation, 14, 285-302.

McDonald, S., Flanagan, S., Rollins, J., \& Kinch, J. (2003). TASIT: A new clinical tool for assessing social perception after traumatic brain injury. Journal of Head Trauma Rehabilitation, 18, 219-238.

Meulenbroek, P., Turkstra, L., \& Bowers, B. (2016). Characterizing common workplace communication skills for disorders associated with traumatic brain injury: A qualitative study. Journal of Vocational Rehabiltation, 44(1), 15-31.

Milders, M., Fuchs, S., \& Crawford, J. R. (2003). Neuropsychological impairments and changes in emotional and social behaviour following severe traumatic brain injury. Journal of Clinical \& Experimental Neuropsychology, 25(2), 157-172.

Mills, A., \& Kreutzer, J. S. (2015). Theoretical applications of positive psychology to vocational rehabilitation after traumatic brain injury. Journal of Occupational Rehabilitation, 1-12.

Njomboro, P., Humphreys, G. W., \& Deb, S. (2014). Exploring social cognition in patients with apathy following acquired brain damage. BioMed Central Neurology, 14 (18).

Nybo, T., Sainio, M., \& Müller, K. (2004). Stability of vocational outcome in adulthood after moderate to severe preschool brain injury. Journal of the International Neuropsychological Society, 10, 719-723.

Power, P. W., \& Hershenson, D. B. (2003). Work adjustment and readjustment of persons with mid-career onset traumatic brain injury. Brain Injury, 17, 1021-1034.

Robertson, I. H. (1996). Goal Management Training: A Clinical Manual. Cambridge: PsyConsult.

Sale, P., West, M., Sherron, P., \& Wehman, P. H. (1991). Exploratory analysis of job separation from supported employment of persons with traumatic brain injury. Journal of Head Trauma Rehabilitation, 6, 1-11.

Sherer, M., Boake, C., Levin, E., Silver, B., Ringholz, G., \& High, W. (1998). Characteristics of impaired awareness after traumatic brain injury. Journal of the International Neuropsychological Society, 4, 380-387.

Stone, V. E., Baron-Cohen, S., \& Knight, R. T. (1998). Frontal lobe contributions to theory of mind. Journal of Cognitive Neuroscience, 1, 640-656.

Struchen, M. A., Clark, A. N., Sander, A. M., Mills, M. R., Evans, G., \& Kurtz, D. (2008). Relation of executive functioning and social communication measures to functional outcomes following traumatic brain injury. Neurorehabilitation, 23, 185198.

Struchen, M. A., Pappadis, R., Sander, A. M., Burrows, C. S., \& Myszka, K. A. (2011). Examining the Contribution of Social Communication Abilities and Affective/Behavioral Functioning to Social Integration Outcomes for Adults With Traumatic Brain Injury. Journal of Head Trauma Rehabilitation, 26(1), 30-42.

Togher, L., McDonald, S., Code, C., \& Grant, S. (2004). Training communication partners of people with traumatic brain injury: A randomised controlled trial. Aphasiology, 18, 313-335

Tyerman, A., Tyerman, R., \& Viney, P. (2008). Vocational Rehabilitation Programmes. In A. Tyerman \& N. King (Eds.), 
Psychological Approaches to Rehabilitation after Traumatic Brain Injury. Oxford: Blackwell, pp. 376-402.

Van Der Horn, H. J., Spikman, J. M., Jacobs, B., \& Van Der Naalt, J. (2013). Postconcussive complaints, anxiety, and depression related to vocational outcome in minor to severe traumatic brain injury. Archives of Physical Medicine \& Rehabilitation, 94(5), 867-874.

Villki, J., Ahola, K., Holst, P., Ohman, J., Servo, A., \& Heiskanen, O. (1994). Prediction of psychosocial recovery after head injury with cognitive test and neurobehavioural ratings. Journal of Clinical Experimental Neuropsychology, 16, 325-338.

Walsh, S., Fortune, D. G., Gallagher, S., \& Muldoon, O. (2014). Acquired brain injury: Combining social psychological and neuropsychological perspectives. Health Psychology Review, 8(4), 458-472.

Watt, N., \& Penn, C. (2000). Predictors and indicators of return to work following traumatic brain injury in South Africa: Findings from a preliminary experimental database. South African Journal of Psychology, 30, 27-37.

Wehman, P. H., Kregel, J., Sherron, P. D., Nguyen, S., Kreutzer, J. S., Fry, R., \& Zasler, N. (1993). Critical factors associated with the successful supported employment placement of patients with severe traumatic brain injury. Brain Injury, 7, 31-44.
Weiss, D. S., \& Marmar, C. R. (1997) The Impact of Event Scale - Revised. In Assessing Psychological Trauma and PTSD (eds J. P. Wilson \& T. M. Keane), New York: Guilford Press, pp. $399-411$.

Wilson, B. A., Alderman, N., Burgess, P., Emslie, H., \& Evans, J. J. (1996). Behavioural Assessment of Dysexecutive Syndrome (BADS). Bury St Edmonds: Thames Valley Test Company.

Yeates, G. N. (2013). Towards the neuropsychological foundations of couples therapy following acquired brain injury (ABI): A review of empirical evidence and relevant concepts. NeuroDisability \& Psychotherapy, 1(1), 108-150.

Yeates, G. N. (2014). Social cognition interventions in neuro-rehabilitation: An overview. Advances in Clinical Neuroscience \& Rehabilitation, 14(2), 12-13.

Yeates, G. N., Milders, M. M., \& Eley, D. (2013) The recognition of faux pas test in an acquired brain injury analysis: Performance and error analysis.Paper presented at the WFNR Neuropsychological Symposium Annual Meeting, Maastricht, Netherlands.

Zigmond, A. S., \& Snaith, R. P. (1983).The hospital anxiety and depression scale. ActaPsychiatricaScandinavica, 67, 361-370. 